\title{
Enhanced photoacoustic spectroscopy sensitivity through intra-cavity OPO excitation.
}

\author{
Adam Polak*a, David J. M. Stothard ${ }^{\mathrm{a}}$ \\ ${ }^{a}$ Fraunhofer Centre for Applied Photonics, Fraunhofer UK Research Ltd, \\ 99 George Street, Glasgow, G1 1RD, UK;
}

\begin{abstract}
We report an optical molecular gas sensor exhibiting high levels of selectivity and sensitivity. The outstanding sensitivity demonstrated by our technology is rooted in a novel combination of photoacoustic spectroscopy (PAS) operated within the cavity of a continuous-wave, intra-cavity Optical Parametric Oscillator (OPO). We exploit the very high circulating field present within the resonant down-converted cavity as the excitation source of the photoacoustic effect, conferring orders-of-magnitude improvement in optical excitation power. Additionally, the wide selectivity of the system arises from the inherent broad tunability and narrow optical linewidth of an OPO.

Here we report the use of this technology for the detection of ammonia $\left(\mathrm{NH}_{3}\right)$ as a simulant target molecule. A 3-D printed miniature PAS cell with microelectromechanical systems based (MEMS) microphone is used for the gas detection. The resonance frequency of the cell was measured at $17.9 \mathrm{kHz}$ with a Q-factor of 9 . The down-converted signal wave resonating within its optical cavity was tuned to $6605.6 \mathrm{~cm}^{-1}$ (corresponding to a strong local $\mathrm{NH}_{3}$ absorption line) through a combination of phase matching and intra-cavity etalon control. The laser was amplitude modulated at the resonance frequency of the PAS cell, producing an average optical excitation power of $\sim 10 \mathrm{~W}$ in the signal arm of the OPO, to induce the photoacoustic effect for only $4 \mathrm{~W}$ of primary diode pump power.

In this work we show detection limit at the level of single parts-per-billion (ppb). Additionally, we will discuss how this technology could be readily refined to potentially demonstrate a sensitivity of tens parts-per-quadrillion.
\end{abstract}

Keywords: Photoacoustic Spectroscopy (PAS), Optical Parametric Oscillator (OPO), Intra-cavity, Gas sensing.

\section{INTRODUCTION}

Detection of explosive materials is one of the major aspects of homeland security and counter-terrorism. Since explosives are usually made from volatile organic compounds (VOCs), gas detection is a vital method to identify threat. Concealment of the source material, low vapour pressures of most VOCs and usually only traces of them in the ambient atmosphere make this detection a challenging task. Various techniques found their application in the detection of explosive materials, including spectroscopic methods, olfactory and chemical sensors as well as nanoscale and thin films sensing ${ }^{1}$. Among many spectroscopic techniques used for explosives detection, Photoacoustic Spectroscopy has also demonstrated its applicability for gas trace detection ${ }^{2-5}$. From within optical methods, this one is of special interest for explosives detection, as VOCs exhibit their most prominent features in mid- and deep-infrared regime ${ }^{6}$, where optical detectors are complex, expensive and often characterised with poor detectivity. Over past years various different embodiments of PAS were demonstrated, regarding both the excitation method (including, but not limited to, laser diodes ${ }^{7}, \mathrm{OPO}^{8}$ and quantum cascade lasers $(\mathrm{QCL})^{2}$ ) as well as the spectrophone (including microphone based ${ }^{4,9}$ and quartz enhanced (QE-PAS) versions $^{2,7}$ ). Independent from the realisation of the photoacoustic system, the signal it produces can be expressed as:

$$
S \propto k \alpha Q P
$$

where $\alpha$ is the molecular absorption of the observed molecules, $\mathrm{Q}$ is the resonant quality factor of the spectrophone, $\mathrm{P}$ is the incident optical excitation power and $\mathrm{k}$ is an constant describing other properties of the instrument ${ }^{10,11}$. Since the signal is directly proportional to these factors, it can be therefore maximised on different routes. The QE-PAS systems exploit the very high $\mathrm{Q}$ of the quartz tuning fork based spectrophones and selection of mid- and deep-infrared excitation

*adam.polak@fraunhofer.co.uk; phone +44(0) 141548 4667; www.fraunhofer.co.uk 
sources aims at the strongest absorption features - especially for VOC's. Increase of the optical power opens another modality for enhanced PAS sensitivity. Although high power infrared lasers are readily available $\left(\mathrm{CO}_{2}, \mathrm{CO}, \mathrm{DF}\right.$ etc. $)$, they are large and usually operate on fixed frequency which makes them not practical for the detection of explosives. Another source of infrared radiation are OPOs and in contrast to the aforementioned lasers, these systems are broadly tuneable. OPOs, in their traditional extra-cavity configuration, require high power pump lasers to bring them to threshold, which makes them similarly impractical for deployable gas detection. However, the intracavity approach (IC-OPO) exploits the high circulating optical field of the laser that brings the parametric effect to the threshold already with several hundred milliwatts of primary pump power ${ }^{12}$. We propose to use the same feature to enhance sensitivity of photoacoustic spectroscopy setup.

In this paper we aim to demonstrate proof-of-concept of ultra-sensitive technique for application in detection of concealed explosives. It exploits the enhancement of the photoacoustic signal by location of spectrophone inside (intracavity to) the oscillating arm of an IC-OPO and use of very high circulating optical field found in this cavity as an excitation source. We describe lab-based project, demonstrating detectivity of ammonia to the single parts-per-billion level. We also propose ways of further refinement of this technique that could lead to the unprecedented at these wavelengths parts-per-quadrillion sensitivity levels.

\section{METHODOLOGY}

\subsection{Optical Parametric Oscillator}

During this work, a standing wave cavity design was chosen for the IC-OPO. Due to the selection of ammonia as the simulant molecule, the signal resonant configuration was built, to target the ammonia absorption lines in near-infrared region. Figure 1 illustrates the schematic diagram of our system.

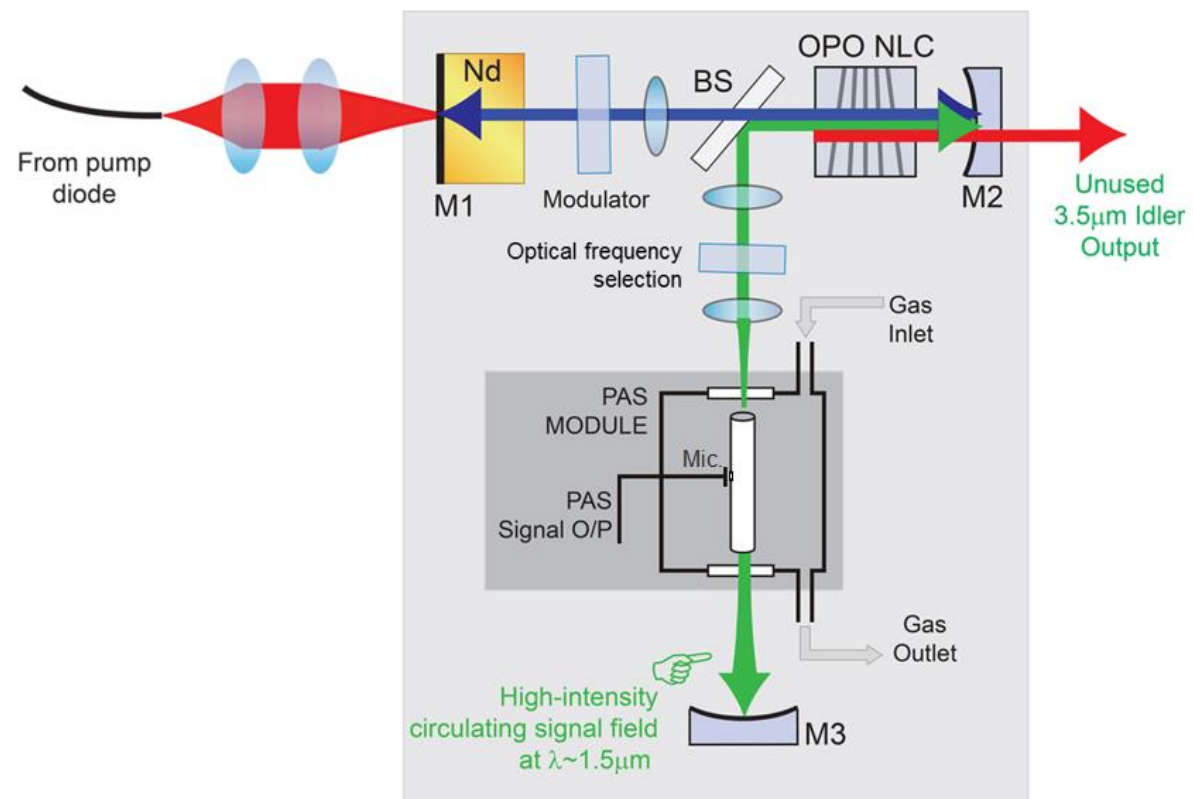

Figure 1. Schematic representation of the signal resonant IC-OPO with an intracavity PAS spectrophone, as developed during this project

The parent laser cavity, oscillating a pump wave at 1064nm, is defined by mirror M1, (a highly-reflecting (HR) dielectric coating applied to the outer surface of a $3 \times 3 \times 1 \mathrm{~mm}^{3}$ a-cut $2 \%$ doped Nd:YVO4 laser gain crystal), and M2 with similar coating. All components in between were anti-reflection (AR) coated at this pump wavelength. The M1 coating was additionally AR coated for $808 \mathrm{~nm}$ diode pump light provided by a $4 \mathrm{~W}, 200 \mu \mathrm{m}$ core size fibre-coupled laser diode (Sheaumann Laser, Part No: HF-808-010W-25C). An acousto-optic modulator (AOM) (Gooch \& Housego, 
Part No: I-QS080-1C10G-4-OS13) was provided in the pump cavity to create amplitude modulation on the continuouswave circulating field.

The nonlinear gain material was a periodically-poled 30x15x1mm3 MgO doped $\mathrm{LiNbO}_{3}$ crystal (PPLN) which featured a fanned-shape quasi-phase matching grating, with period ranged from $27-32 \mu \mathrm{m}$ over the $15 \mathrm{~mm}$ axis of the crystal. In the developed embodiment, the crystal was not temperature stabilised as the operation wavelength was stabilised by presence of an etalon in the resonant arm of the OPO. The signal cavity was defined by mirror M2 (HR at the signal as well as the pump wavelength), dichroic beam splitter - BS (AR coated on both sides for the pump wave; HR on the inner surface for

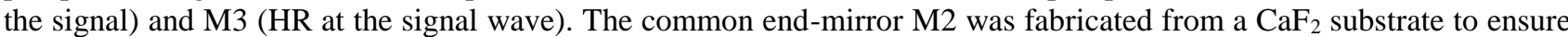
high transmission at deeper IR wavelengths; it was also coated for good transmission at the idler bandwidth which exited through this mirror after being generated in the nonlinear material. The mid-infrared output-coupled idler which is usually of interest, it was unused in this work.

Due to the fact that at the frequency of the ammonia absorption features, the phase matching gain bandwidth of the PPLN crystal is approximately $300 \mathrm{GHz}\left(\sim 10 \mathrm{~cm}^{-1}\right)$, some form of additional frequency selectivity was required in the signal arm of the OPO. During this work we have implemented two forms of frequency selective devices: a $333 \mathrm{GHz}$ free spectral range (FSR), 20\% reflectivity at the signal wavelengths range air spaced etalon (ASE) and a $4.155 \mathrm{~mm}$ long uncoated solid silicon etalon ( 10GHz FSR) with surfaces exhibiting a Fresnel-reflectivity of $30 \%$, due to the high refractive index of silicon. Both etalons were supplied by LightMachinery Inc., Canada.

\subsection{Photoacoustic spectrophone}

In this proof-of-concept study we have prepared a 3D-printed cell modelled on the design proposed by Bauer at al. ${ }^{9}$. We have chosen this composition as its miniature nature (total length of the acoustic cavity is only 10mm) allowed for compact OPO design with the intracavity location of the PAS cell. Figure $2 \mathrm{a}$ shows cross-section of the cell design and Figure $2 \mathrm{~b}$ the final printed one.

a)

b)

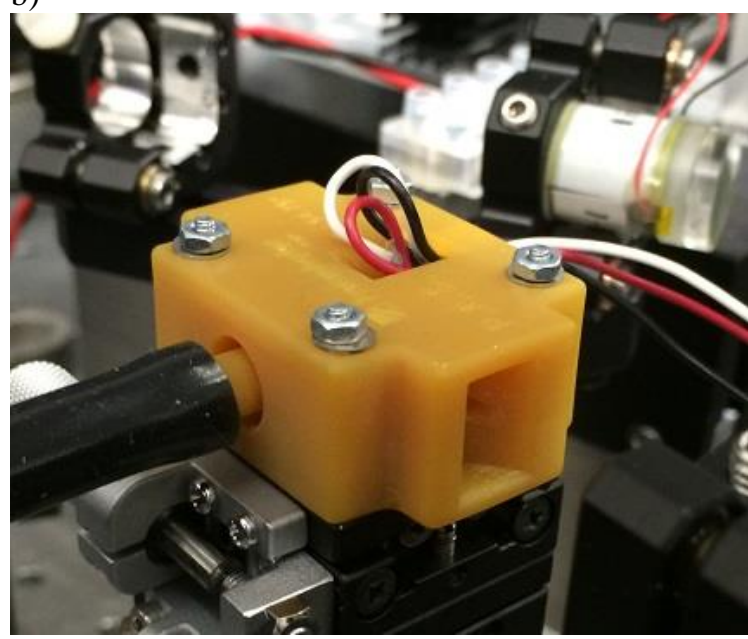

Figure 2. The spectrophone used for this study: cross-section design (a) and fully integrated in the optical setup (b)

In our system, the excitation beam was coupled to the cell from the open space laser, therefore originally Brewster angled windows were designed, but they were not mounted on the ready cell to minimise potential optical losses. The ammonia samples prepared for the experiment were in a form of gas mixture of $100 \mathrm{ppm}$ of $\mathrm{NH}_{3}$ in nitrogen (balance). The resonance frequency of the cell was calculated based on formula (2) for resonance condition of open pipes

$$
f_{c}=\frac{c}{2 l}
$$

where $\mathrm{c}$ is the speed of sound in given medium and 1 is the length of the resonator. Since nitrogen was the main compound in the mixture, the resonance was calculated with assumption of nitrogen as the gaseous medium, resulting in $f_{c}=17.6 \mathrm{kHz}$. To match this resonance we used an Invensense INMP504 ultra-miniature analogue MEMS microphone, with relative 
acoustic response ranges from $100 \mathrm{~Hz}$ up to $20 \mathrm{kHz}$. The signal from the microphone was fed to the Lock-in Amplifier (Stanford Research Systems, Model: SR830 DSP) which was referenced by the same signal generator that was driving the acousto-optic modulator.

\subsection{Experimental procedure}

The aforementioned gas mixture used during this study was delivered from high pressure cylinder. During the alignment procedures and cell characterisation the gas was continuously flowing through the cell at the flow rate of $130 \mathrm{ml} / \mathrm{min}$. For the detection sensitivity test, gas was sampled with a $50 \mathrm{ml}$ volume syringe and diluted with air to achieve desired concentration. Although this method may not be highly accurate, due to losses to the syringe walls, it allows to demonstrate the sensitivity levels with acceptable precision.

\section{RESULTS}

\subsection{Power performance}

Since the main part of this innovation comes through the exploitation of the high optical field oscillating in the resonant cavity of the OPO, the power performance of built system was verified prior introduction of PAS setup. In Figure 3 the extracted idler power is shown as a function of incident diode pump power. We can see that at maximum incident $4 \mathrm{~W}$ of diode pump power, $220 \mathrm{~mW}$ of idler power at a wavelength of $3.3 \mu \mathrm{m}$ is obtained through M2. Given the quantum defect between the idler and signal plus the fact that the signal and idler are generated in both directions, it is possible to work out the total down-converted power. It can be seen that $\sim 1.45 \mathrm{~W}$ of power was down-converted from the circulating $1064 \mathrm{~nm}$ pump to the signal and idler fields.

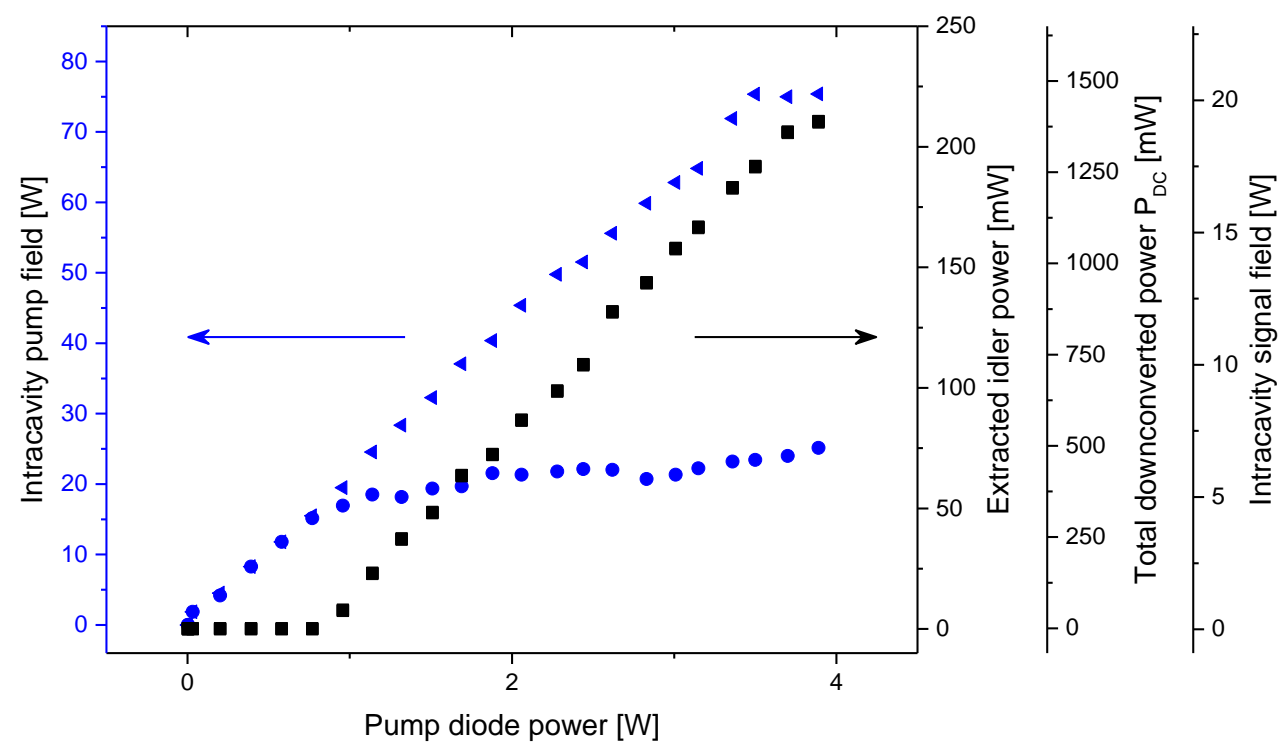

Figure 3. Power characteristics of constructed OPO where blue triangles represent intracavity pump field with OPO not working, blue circles depict the same with OPO working and black squares represent extracted idler power, total downconverted power and intracavity signal field on their respective axes

Assuming a round trip loss of 5\% (which is most probably estimated in excess) the total optical field contained within signal cavity can be calculated using the expression:

$$
P_{s}=\left(\frac{\lambda_{i}}{\lambda_{s}} \cdot 2 . P_{\text {idler_ex }}\right) \frac{1}{\beta_{s}}
$$

where $\lambda_{\mathrm{i}}$ and $\lambda_{\mathrm{s}}$ are the respective wavelengths of the idler and signal, $\mathrm{P}_{\text {idler_ex }}$ is the extracted idler power (taking into account extraction loss) and $\beta$ s is the signal cavity round-trip loss. Therefore $220 \mathrm{~mW}$ of extracted idler power gives a circulating signal power of $19.2 \mathrm{~W}$ available as the excitation of the photoacoustic effect. It must be noted that during this 
characterisation none of the frequency selective elements was present in the cavity. Additionally, this test was done without the amplitude modulation enabled, which significantly affects the average power of the system. With all the components in the system approximately $8 \mathrm{~W}$ of the oscillating signal power that was available as excitation during this experiment.

\subsection{Spectrophone characterisation}

Printed and assembled spectrophone was placed at the waist of the circulating signal wave of the OPO tuned to operate on $1531 \mathrm{~nm}$ (to coincide with the $6528.8 \mathrm{~cm}^{-1}$ absorption line of ammonia).The amplitude modulation (obtained with use of $\mathrm{AOM}$ ) was tuned between $5 \mathrm{kHz}$ and $25 \mathrm{kHz}$ and with continuous flow of the gas mixture though the spectrophone, its output signal was monitored by the Lock-in Amplifier. The resulting characteristics are presented in Figure 4.

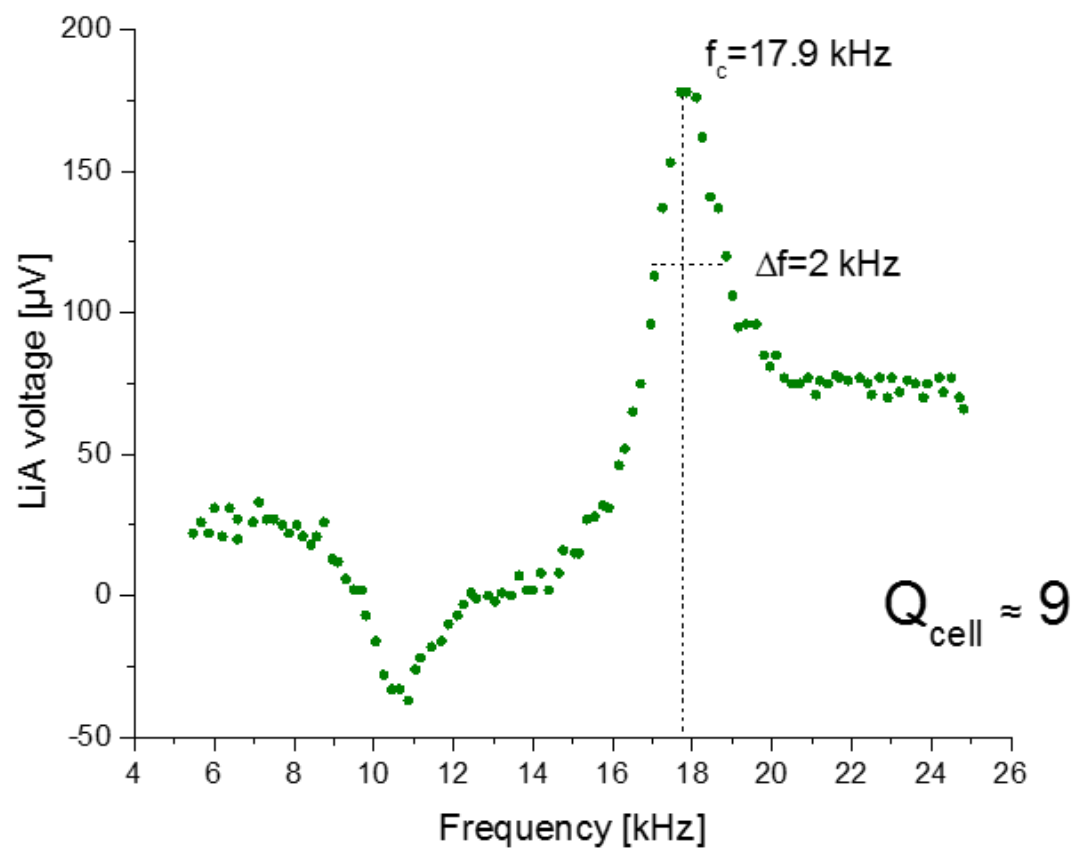

Figure 4. Characteristic of the PAS spectrophone in the intracavity position as the function of the modulation frequency

The test results illustrated in Figure 4 indicate resonance frequency at $f_{c}=17.9 \mathrm{kHz}$ which correlate with the theoretical calculations. Knowing the frequency characteristic of the resonator we can derive its quality factor based on the formula (4):

$$
Q=\frac{f_{c}}{\Delta f}
$$

where $\Delta \mathrm{f}$ is the full width at half maximum (FWHM) bandwidth of the damped resonator. Based on the measured characteristic, the $2 \mathrm{kHz}$ bandwidth results in the $\mathrm{Q}$ factor in the region of 9.This value is approximately 3 times smaller than presented by Bauer et al. ${ }^{9}$ and we expect this to be caused by the surface roughness of the resonator affecting the oscillating sound wave.

\subsection{Sensitivity results}

For the sensitivity testing, the OPO was now tuned to $1514 \mathrm{~nm}$ in order to coincide with stronger absorption line of ammonia on $6605.6 \mathrm{~cm}^{-1}$. The continuous flow of ammonia was also replaced by the discrete injections with the syringe as described in Section 2.3. Figure 5 illustrates the photoacoustic signal measured during this test as a function of the ammonia concentration.

As demonstrated, the system exhibits levels of sensitivity on the parts per-billion scale. This corresponds with the detectability levels reported by Bauer et al. ${ }^{9}$ (upon whose spectrophone design was based) when one accounts for the enormous improvement brought by the intracavity excitation field. In their experiment, Bauer's group found their sensor 
to be limited to $2.5 \mathrm{ppm}$ for $22 \mathrm{~mW}$ excitation power, and no averaging. Given the similar absorption coefficients of the $\mathrm{C}_{2} \mathrm{H}_{2}$ (acetylene) sample gas they were using and the $\mathrm{NH}_{3}$ which we used, and assuming a circulating signal-wave power of $\sim 8 \mathrm{~W}$, we would therefore expect a sensitivity improvement of $\sim 360$ times, placing our sensitivity limit in the region of a $1 \mathrm{ppb}$ - as indeed we observed.

$\mathrm{NH} 3$ concentrationtests with $50 \mathrm{ml}$ of gas tested @ $6605.60 \mathrm{~cm}-1$

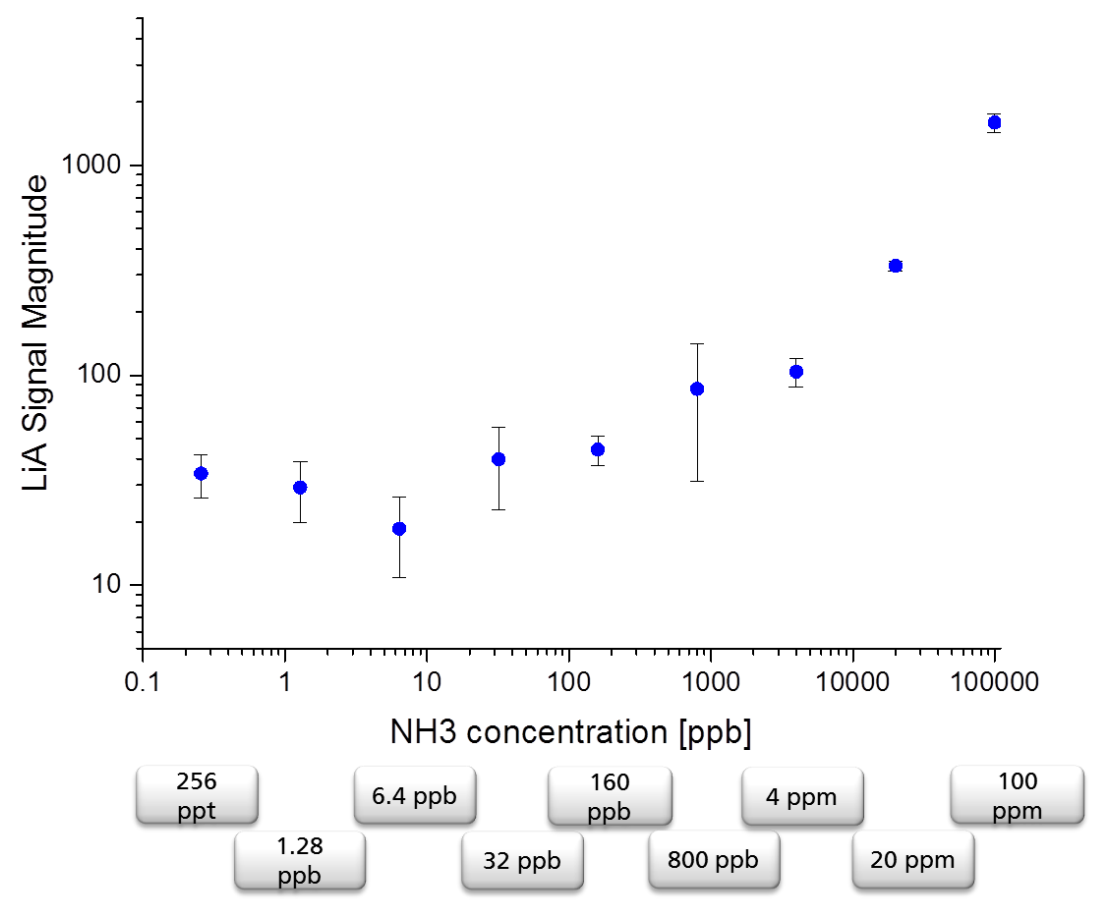

Figure 5. Sensitivity of the system to ammonia on absorption line $6605.6 \mathrm{~cm}^{-1}$

\section{FUTURE WORK}

Results achieved in this study demonstrated significant improvement of photoacoustic spectroscopy sensitivity, resulting from the intracavity location of the spectrophone. However, there are still significant improvements that could be done to bring the detectivity of our system to even lower levels. In this work we focused on the advantages coming from very high optical power of the oscillating intracavity field, yet, as demonstrated in formula (1), this is only one of the factors that can affect the sensitivity of the PAS signal. In fact each of these parameters qualify as a potential route for improvements. The absorption coefficient for most of the compounds of interest increases towards longer wavelengths. Use of idler wave produced by OPO will enable detection in mid-infrared range that on average can bring approximately tenfold sensitivity enhancement. The most prominent route is the increase of the resonator's quality factor. In this work we used a miniature cell exhibiting very low $\mathrm{Q}$ factor of less than 10. Replacing this by a tuning fork based spectrophone (QE-PAS) characterised by approximately Q-factor of $10000{ }^{10}$ would bring about three orders of magnitude detectivity levels boost. We do not plan additional increase of power as a route for further improvements. Although IC-OPOs can be pumped by tens of Watts of primary diode power, this causes heat extraction problems and make these systems bulky and difficult to translate into deployable devices. On the other hand, we perceive that the change of the oscillating filed (from signal to idler) results in the effective drop of the optical power, caused by the quantum defect between these waves. At last, we recognise the benefits of the lock-in averaging, where output signal could be potentially averaged by tens of seconds resulting in further sensitivity improvements. In Table 1 we demonstrate these potential refinements of our system, with identification of quantified impact of each step. The result of presented here proof-of-concept study (1ppb) is used as a basis of these predictions, leading to the detectivity levels of single parts-per-quadrillion, with all the improvements implemented. 
Table 1. Summary of the predicted improvements, along with quantification of their magnitude; the sensitivity developed in this project is used as a base line to these projections

\begin{tabular}{|c|c|c|c|c|}
\hline Item & $\begin{array}{c}\text { Current (PAS } \\
\text { spectrophone at } \\
\text { signal wavelength) }\end{array}$ & $\begin{array}{c}\text { Refinement (QE-PAS } \\
\text { spectrophone at idler } \\
\text { wavelength) }\end{array}$ & $\begin{array}{c}\text { Improvement } \\
\text { in SNR }\end{array}$ & $\begin{array}{c}\text { Sensitivity limit } \\
\text { (starting from } \\
\text { current 1ppb) }\end{array}$ \\
\hline$\alpha$ & $\begin{array}{c}\text { Signal absorption } \\
\text { coefficient }\end{array}$ & $\begin{array}{c}\text { Idler absorption } \\
\text { coefficient }\end{array}$ & $10 \mathrm{x}$ & $100 \mathrm{ppt}$ \\
\hline $\mathrm{Q}$ & $\sim 10$ & $\sim 10,000$ & $1000 \mathrm{x}$ & $100 \mathrm{ppq}$ \\
\hline $\mathrm{P}$ & $\sim 8 \mathrm{~W}$ & $\begin{array}{c}\sim 4 \mathrm{~W} \text { (power drop due to } \\
\text { estimated signal idler } \\
\text { quantum defect) }\end{array}$ & $0.5 \mathrm{x}$ & $200 \mathrm{ppq}$ \\
\hline $\mathrm{k}$ & No time averaging & Time averaging & $100 \mathrm{x}$ & $\sim 2 \mathrm{ppq}$ \\
\hline
\end{tabular}

\section{CONCLUSIONS}

In this paper we demonstrate proof-of-concept study as a route to ultra-sensitive molecular detection based upon a novel embodiment of photoacoustic spectroscopy. To this end, we succeeded in our primary goal of representing the sensitivity improvements of photoacoustic techniques achieved by implementation of the very high circulating field found within the cavity of an optical parametric oscillator. Even though the power circulating in this cavity was not optimised, we showed that our approach is able to increase the excitation power to approximately $10 \mathrm{~W}$, leading to detectivity levels of single parts-per-billion. These results demonstrate about 360 times improvement in sensitivity to the comparable system with extra-cavity laser diode excitation source. Lastly, we also demonstrated possible routes for rectification of this proof-ofconcept setup that may lead to sensitivity of the signal improved to ppq levels.

\section{ACKNOWLEDGEMENTS}

This project was funded under the Innovative Research Call in Explosives and Weapons Detection 2016. This is a CrossGovernment programme sponsored by a number of Departments and Agencies under the UK Government's CONTEST strategy in partnership with the US Department of Homeland Security, Science and Technology Directorate. This work was also supported by an Industrial Fellowship from the Royal Commission for the Exhibition of 1851.

\section{REFERENCES}

[1] Caygill, J. S., Davis, F. and Higson, S. P., "Current trends in explosive detection techniques," Talanta 88, 14-29 (2012)

[2] Bauer, C., Willer, U., Lewicki, R., Pohlkötter, A., Kosterev, A., Kosynkin, D., Tittel, F. K. and Schade, W., "A Mid-infrared QEPAS sensor device for TATP detection," Journal of Physics: Conference Series 157(1), 1-6 (2009)

[3] Harren, F. J. M., Bijnen, F. G. C., Reuss, J., Voesenek, L. A. C. J. and Blom, C. W. P. M., "Sensitive Intracavity Photoacoustic Measurements with a CO2 Waveguide Laser," Applied Physics B 50(2), 137-144 (1990)

[4] Khalil, A., Gondal, M. and Al-Suliman, N., "Resonant photo-acoustic detection of carbon monoxide with UV Laser at 213 nm," Applied Physics B 103(2), 441-450 (2011)

[5] Miklós, A., Hess, P. and Bozóki, Z., "Application of acoustic resonators in photoacoustic trace gas analysis and metrology," Review of Scientific Instruments 72(4), 1937-1955 (2001)

[6] Janni, J., Gilbert, B. D., Field, R. and Steinfeld, J. I., "Infrared absorption of explosive molecule vapors," Spectrochimica Acta Part A: Molecular and Biomolecular Spectroscopy 53(9), 1375-1381 (1997)

[7] Serebryakov, D., Morozov, I., Kosterev, A. and Letokhov, V., " Laser microphotoacoustic sensorof ammonia traces in the atmosphere," Quantum Electronics 40(2), 167-172 (2010)

[8] Herpen, M. v., Bisson, S., Ngai, A. and Harren, F., "Combined wide pump tuning and high power of a continuouswave, singly resonant optical parametric oscillator," Applied Physics B Lasers and Optics 78, 281-286 (2004) 
[9] Bauer, R., Stewart, G., Johnstone, W., Boyd, E. and Lengden, M., "3D-printed miniature gas cell for photoacoustic spectroscopy of trace gases," OPTICS LETTERS 39(16), 4796-4799 (2014)

[10] Patimisco, P., Scamarcio, G., Tittel, F. K. and Spagnolo, V., "Quartz-Enhanced Photoacoustic Spectroscopy: A Review," Sensors 14, 6165-6206 (2014)

[11] Elia, A., Lugarà, P. M., Franco, C. D. and Spagnolo, V., "Photoacoustic Techniques for Trace Gas Sensing Based on Semiconductor Laser Sources," Sensors 9, 9616-9628 (2009)

[12] Stothard, D. J. M., "Practical Continuous-Wave Intracavity Optical Parametric Oscillators," in [Advances in Optical and Photonic Devices], K. Y. Kim, Ed., InTech, 293-328 (2010) 